\title{
Tracking the Randomized Rollout of a Veterans Affairs Opioid Risk Management Tool: A Multi- method Implementation Evaluation Using the Consolidated Framework for Implementation Research
}

Sharon McCarthy ( $\nabla$ sharon.mccarthy@va.gov)

VA Pittsburgh Healthcare System - Highland Drive Campus: VA Pittsburgh Healthcare System https://orcid.org/0000-0002-7299-5129

\section{Matthew Chinman}

VA Pittsburgh Healthcare System - Highland Drive Campus: VA Pittsburgh Healthcare System

\section{Shari Rogal}

VA Pittsburgh Healthcare System - Highland Drive Campus: VA Pittsburgh Healthcare System Gloria Klima

VA Pittsburgh Healthcare System - Highland Drive Campus: VA Pittsburgh Healthcare System

Leslie Hausmann

VA Pittsburgh Healthcare System - Highland Drive Campus: VA Pittsburgh Healthcare System

Maria Mor

VA Pittsburgh Healthcare System - Highland Drive Campus: VA Pittsburgh Healthcare System

Mala Shah

VA Pittsburgh Healthcare System - Highland Drive Campus: VA Pittsburgh Healthcare System Jennifer Hale

VA Pittsburgh Healthcare System - Highland Drive Campus: VA Pittsburgh Healthcare System

Hongwei Zhang

VA Pittsburgh Healthcare System - Highland Drive Campus: VA Pittsburgh Healthcare System

Adam Gordon

VA Salt Lake City Health Care System

Walid Gellad

VA Pittsburgh Healthcare System

Research

Keywords: Veterans Affairs, Consolidated Framework, Engaging 
Posted Date: August 30th, 2021

DOl: https://doi.org/10.21203/rs.3.rs-826458/v1

License: (c) (i) This work is licensed under a Creative Commons Attribution 4.0 International License. Read Full License 


\section{Abstract}

\section{Background}

The Veterans Health Administration (VHA) developed the Stratification Tool for Opioid Risk Mitigation (STORM) dashboard to assist VHA clinicians in identifying Veterans at risk for adverse opioid overdose or suicide-related events. In 2018, a national policy was implemented requiring providers at all VHA facilities to complete case reviews of Veterans identified by STORM as very high risk for adverse events.

Nationally, facilities were randomized by the type of oversight required when sufficient case reviews were not completed and also by the timing of an increase in the number of required case reviews.

As part of a comprehensive assessment of this policy intervention, we aimed to 1) identify barriers and facilitators to implementing case reviews as required in the policy; 2 ) assess variation across the four arms of the study; and 3) evaluate associations between facility characteristics and implementation barriers and facilitators.

\section{Methods}

Using the Consolidated Framework for Implementation Research (CFIR), we developed a semi-structured interview guide to examine barriers to and facilitators of implementing the STORM policy. Staff from 40 purposefully selected facilities who were involved in implementation were invited to participate in telephone interviews. Interview transcripts were coded and then organized into memos, which were numerically rated using the -2 to +2 CFIR rating system for each construct. Descriptive statistics were used to evaluate the mean ratings on each CFIR construct, the associations between ratings and study arm, and three facility characteristics (size, rurality, and level of academic detailing) associated with CFIR ratings. We used the mean CFIR rating for each site to determine which constructs differed between the sites with highest and lowest overall CFIR scores, and these constructs were described in detail.

Results

Interviews with 78 staff at $39 \mathrm{VHA}$ facilities identified a slightly positive $(+0.2)$ overall mean CFIR rating. CFIR ratings were not significantly different between the four study arms, nor associated with facility characteristics. Overall, two important barriers to implementation were CFIR constructs Access to knowledge and information and Evaluating and reflecting. Having time to complete the reviews was a pervasive barrier. Sites with higher overall CFIR scores showed three important facilitators: Leadership engagement, Engaging, and Implementation climate.

\section{Conclusion}

Although there was variability in implementation barriers and facilitators across facilities, these were unrelated to study arms and facility characteristics. Leadership, resources, and overall implementation climate were the strongest facilitators of policy implementation. 


\section{Contributions To The Literature:}

- In this nationwide implementation of a policy requiring the review of high-risk patients for opioid adverse events, primary facilitators were leadership engagement, resource availability (especially time), appropriately engaged individuals and positive implementation climate.

- The CFIR memo methodology provided useful comparison of sites rated higher and lower overall on specific constructs functioning as barriers and facilitators, displayed in a joint display of both qualitative and quantitative outcomes.

- The findings suggest the limits to using official policies promising low level consequences as a strategy to overcome implementation barriers, as randomization to consequence or no consequence showed no differences.

\section{Background}

Many health systems, including the Veterans Health Administration (VHA), have taken proactive, multifaceted approaches to mitigate the harms of opioids in the United States. As part of these continuing efforts, VHA developed the Stratification Tool for Opioid Risk Mitigation (STORM), a suite of provider-facing electronic reports which use a predictive model to identify patients at risk for opioid or suicide-related events [1]. Updated nightly, the STORM reports provide an estimated risk level for all Veteran patient, as well as additional information to assist clinicians to apply appropriate risk mitigation strategies tailored to individual patient risk factors and needs.

In March of 2018, VHA released a national policy notice requiring that all facilities begin conducting "case reviews" of Veterans determined to be at highest risk based on the STORM model. Completing these case reviews entailed using a data tool, such as the STORM dashboard, to evaluate risk and determine the utility of providing risk mitigation strategies (e.g., referral to a pain specialist, prescription of naloxone). Completed case reviews and actions taken by the clinician(s) were documented using a standardized note in the VA electronic medical record.

The implementation of this policy notice was randomized in an effort to meet continuous improvement goals. [1,2] Half of VHA facilities were randomly assigned to receive a version of the policy notice that indicated additional oversight would be required if, after six months, the site did not achieve a case review completion rate of $97 \%$, calculated as the number of completed case reviews of those Veterans identified by the STORM model as high risk [2,3]. In addition, using a stepped-wedge design, VHA facilities were further randomized to require case reviews for an expanded group of Veterans (moving from top 1\% of those at risk to top 5\%) at either 9 or 15 months after the notice was released. This created four study arms, varying by oversight/no oversight and length of time before the number of case reviews required would increase. This design allowed for examination of the effect of requiring case reviews for the top $1 \%$ versus $5 \%$ of high-risk Veterans and the impact of requiring additional oversight for facilities that failed to meet a target goal on case review completion efforts. 
The first phase of a national evaluation of this policy notice identified specific strategies associated with improved case review completion and found that being randomized to receive additional oversight did not impact the number or type of implementation strategies used to complete case reviews [4]. In the second phase of evaluation, described here, we conducted over 80 qualitative interviews representing 40 VHA sites to examine barriers and facilitators to implementing the case review policy. The interview protocol was developed using the Consolidated Framework for Implementation Research (CFIR) (https://cfirguide.org/) and the resulting memos summarizing each site's experience were rated using the CFIR rating strategy $[5,6,7]$. This phase of evaluation aimed to 1 ) identify the barriers to and facilitators of implementing required case reviews; 2 ) assess variation in barriers and facilitators across the four study arms, and 3) evaluate the associations between CFIR ratings and three facility characteristics: facility size, rural or urban location, and level of support provided through academic detailing. VHA is one of few healthcare systems with the infrastructure to support an evaluation of a nation-wide policy implementation when sites are given substantial discretion as to how to set up their local implementation efforts. Thus, these data can inform future large-scale policy-driven implementation efforts.

\section{Methods}

This study was reviewed and approved by the VA Pittsburgh Health Care System's Institutional Review Board. An overview of the STORM evaluation protocol and the results of implementation strategies assessments were previously published [2,3].

\section{Sample Design and Participant Recruitment}

Within each of the four study arms, we purposefully sampled 10 sites, using baseline performance on a national VA metric called the Opioid Therapy Guideline Adherence Metrics (OTG), which measures the extent a facility is following VHA national best practices for opioid treatment. Our aim with this sampling strategy was to interview sites with varying levels of baseline performance on opioid risk mitigation strategies. A statistician not associated with the interview process ranked sites in each arm based on their performance on the OTG metrics, and we selected 5 sites with the highest OTG scores and 5 with the lowest from each of the four study arms. If a site could not be reached or declined to participate, we moved to the next site on the list for that arm.

We contacted the individual who served as the STORM point of contact (POC) at the selected sites by email, with phone and direct-messaging follow ups, and we invited them to participate in one 45-minute interview. A total of 63 sites were contacted to obtain the target sample of 40 sites. During the interview the POC was asked to suggest one or two other individuals who could comment on the policy implementation at their site, and these individuals were then invited to participate. A total of 82 participants at 40 sites were interviewed, with 9 sites having 1 interview, 21 sites with 2 interviews, and 9 sites with 3 interviews. Two interviews could not be used due to poor audio quality and one site was dropped because they had not started implementation at the time of the interviews. 
The interview guide was developed using CFIR, a meta-theoretical framework for evaluating the factors that influence implementation efforts [5,6,7]. CFIR consists of 32 constructs that influence implementation, organized into five domains: implementation process, characteristics of the clinical intervention being implemented (in this case, the case reviews), characteristics of individuals implementing the clinical intervention, characteristics of the implementing organization (called inner setting), and the impact of forces outside of the organization (called outer setting). CFIR has an existing interview guide that includes questions about all 32 constructs and is designed to be tailored for individual evaluations. As such, it was especially appropriate for this complex implementation effort [8].

Sixteen of the most relevant constructs within four CFIR domains were selected for the interview guide in this study (see Table 3). Data from the individual characteristics domain was not collected because we believed that factors at larger ecological levels were more critical, and asking about all domains would make the interview burdensome. Two research assistants experienced in qualitative interviews were trained to administer the interview, and the interview was piloted with clinicians from two facilities outside the study sample. All interviews were conducted by phone, recorded, transcribed, and validated by trained research staff. Interviews lasted approximately 45 minutes and were conducted from March 2019 through August 2019, approximately one year after the policy was released. Participants provided demographic information, including gender, time in VHA, time in their current role, type of training and clinical practice, and involvement with completing case reviews.

\section{Interview Coding and Memo Development:}

A qualitative content analysis approach was followed [9], using the CFIR constructs as the structure. The coding was approached both deductively using CFIR constructs and inductively as codes emerged from the data [9]. Following transcription and transcript verification, a codebook for the interviews was developed by an experienced qualitative coder (GK). The codebook was reviewed by the research team for completeness, and the coder coded the first 35 interviews using Nvivo 12 software. A second coder (MS) was trained to apply the codes in the codebook to the transcripts and double coded five interviews. Three authors experienced in qualitative research (SM, GK, MS) met to review the double coded interviews and resolve any differences by consensus, adapting the codebook as needed and reviewing previous interviews to maintain consistency. The main coder continued to code interviews, with the secondary coder double coding every fifth interview to prevent drift in coding. All discrepancies were addressed by the qualitative team and significant questions were raised to the entire research team for resolution $[5,6,10]$.

Individual interviews from a single site were combined to create a "memo"-text from all interviews at a site organized by CFIR construct-a CFIR analysis technique used to provide a more complete view of the whole site. The memo approach includes a rating system whereby each individual CFIR construct is rated on a scale of $-2,-1,0,+1,+2$, with negative valence indicating the construct represented a barrier to implementation, and positive valence indicating the construct was a facilitator (https://cfirguide.org/). Six members of the research team were trained in the rating process following CFIR guidelines, and three 
teams of two reviewed site memos and rated the constructs using standard CFIR guidance and exemplars of each category. Each team met to achieve consensus after independently rating constructs for a memo. The entire group of raters also met regularly to discuss any uncertainty in rating and to maintain consistency. As a further check for consistency, an experienced CFIR rater (SM) rated two memos from each team and a comparison showed a high degree of consistency and adherence to CFIR rating guidance. Over time, teams were varied such that coding was done by different partners, to assure continued consistency and prevent team drift in rating. In addition to the memo rating, the first author and one other author (GK) reviewed all the memos and interviews qualitatively, as well as the coded data results (codes within the constructs), and the research team met regularly to discuss recurring themes, both within the constructs and across constructs.

\section{Study arms and facility characteristics}

Study arm was operationalized using two dummy variables: one for the type of policy memo received (standard vs. increased oversight), and one for the timing of the increase in case review requests (early vs. late). Facility-level variables included the number of academic detailing visits, a measure of rurality, and a measure of facility complexity (an algorithm that takes into account patient risk, number and breadth of available specialists, intensive care unit availability, and teaching and research activities)\{2,3\}. Academic detailing is a defined support bundle provided by pharmacists within VHA who help clinicians improve prescribing using training, problem-solving, and data feedback, and was used to operationalize training and support for implementing case reviews.

\section{Analysis}

We used descriptive statistics to characterize the responding providers and sites and to compare the 39 participating sites to facilities where no interviews were conducted. Consistent with the qualitative content analysis, the full set of interview data was explored deductively using coded CFIR constructs, as well as inductively to explore whether any additional barriers or facilitators might emerge that did not fit into the CFIR constructs. Research team members who read and coded the site memos also met regularly to discuss important overall themes in the data.

For each facility, we computed a total CFIR score as the mean summary rating across all 16 constructs (the individual ratings from -2 to +2 for each construct) as well as the proportion of constructs with positive, negative, and zero ratings. We tested the associations between the 16 construct ratings and four study arms, baseline OTG level, and facility characteristics using Kruskal-Wallis tests to allow for nonnormality of the ratings. We identified sites in the top and bottom quartile of CFIR ratings based on the 10 sites with the highest and the 10 sites with the lowest mean CFIR scores. Then, for each of the $16 \mathrm{CFIR}$ constructs, we calculated difference scores by subtracting the mean of that construct in the bottom quartile from the mean of that construct in the top quartile. This allowed for an examination of which CFIR constructs contributed the most to the differences between high and low quartile sites. 
The qualitative and quantitative data were combined using convergent parallel mixed methods [11]. These methods allow for the integration of quantitative data (the difference score for each CFIR construct by high and low quartile) and qualitative data (CFIR interview quotes) that are collected and analyzed separately and in parallel. We integrated those findings using a joint display, a technique that visually combines qualitative and quantitative results to draw out new insights [11].

\section{Results}

The 39 facilities targeted for interviews were similar in terms of facility characteristics to non-interview sites (Supplemental Table 1). The 78 interview participants from these 39 sites were majority women $(58 \%)$ and covered a range of professional disciplines. Most participants had 1 to 5 years of experience in their current roles and were leaders in the STORM implementation efforts at their facility (Table 1). Six facilities were rural and 33 were urban, although many urban facilities have outpatient clinics in more rural locations. Geographically, 8 sites were from the Northeast, 9 were from the Midwest, 7 were from the West and 15 were from the South.

CFIR ratings and associations between CFIR ratings and study arm, OTG, and facility characteristics. Means for each of the CFIR construct ratings across the 39 facilities and randomization arm are listed in Table 2. A positive score indicates the construct is facilitative for implementation, and a negative score indicates the construct is more of a barrier to implementation.

The CFIR ratings for each of the 16 CFIR constructs, the total CFIR scores, and the proportion of positive scores were not significantly different between the four randomization arms (Table 2). For example, overall, the mean CFIR rating in the Engaging construct was 0.6 across all 39 facilities, with a score of $0.6,0.9,0.5$, and 0.4 across facilities in Arms 1 through 4, respectively $(P=0.71)$. Across all constructs and facilities, $40 \%$ of the ratings were positive ( +1 or +2$), 30 \%$ were neutral, and $30 \%$ were negative $(-1$ or -2$)$, with no statistically significant difference across study arms.

There were also no significant differences based on baseline OTG score, oversight vs no oversight and early vs late increase in case reviews (Additional File 1 There was no association between the 16 CFIR construct scores and three facility characteristics: medical center complexity, rural vs urban, and top quartile of academic detailing provided vs other quartiles (Additional File 2).

\section{Mixed methods results with joint display}

Table 3 presents quotes that illustrate each construct as a barrier and as a facilitator and shows the distribution of ratings for 39 sites as well as the difference score on each CFIR construct rating between facilities in the highest and lowest quartiles of overall CFIR scores.

While most constructs functioned as both barriers and facilitators across the sample, there were some domains where facilities had more negative or positive ratings. For example, Evidence Strength and quality was generally a barrier, with positive ratings $(+1)$ at only six sites. Overall, comments show little 
awareness of research or personal evidence to support the completion of case reviews. The Peer pressure construct had only 13 positive ratings(+1) (33\%), and comments showed little evidence that this was either a barrier or facilitator, suggesting little interest in or awareness of how other medical centers nationally were doing on the STORM measures.

Patient needs and resources and Tension for change were generally perceived positively. Patient needs and resources was rated at nearly all sites as neutral $(n=19)$ or positive $(n=15)$, and was rated negatively at only four sites. Likewise, 29 of 39 sites $(74 \%)$ described positive, though moderate (no sites were rated +2) Tension for change, indicating a belief in a clear need for attention to opioid prescribing.

Access to knowledge and information was generally perceived to be an implementation barrier; only 17 of 39 sites rated this positively, and many quotes described a lack training or a lack of awareness of training. Similarly, Reflecting and evaluating was only rated moderately positively $(+1)$ by 6 sites, and many comments described little or confusing feedback on their progress.

The three CFIR constructs with the largest difference scores between the top and bottom CFIR quartiles are Leadership engagement, Engaging, and Implementation climate. Though Networks and Communications had a higher difference score (1.6) than Available resources (1.3), the latter was repeatedly identified in the qualitative analysis and by the qualitative team review as an important determinant of implementation and was therefore included in further analyses. These four constructs are described in more detail below.

\section{Leadership engagement}

The Leadership engagement construct had the largest difference score of 2.1, reflecting a high difference in mean rating between top and bottom quartile facilities (1.2 vs. -0.9). In other words, lack of leadership engagement was a notable barrier for low quartile sites, shown in the following quote where explaining the case review process to leadership is both frustrating and time consuming. When asked "What level of endorsement or support have you seen or heard from leadership?", the team leader commented: "I've heard none...we've had to advocate for what we needed... I've been questioned, like, 'Why do so many people need to be in the room?' Like 'Why do you need more than one doctor to do that kind of work?' And it just says to me, 'Oh, my God, you don't appreciate the complexity of this work'." Asked the same question about level of support, one participant in a low quartile site simply said: "I haven't heard any."

In contrast, facilities with overall high mean CFIR scores described supportive leadership as facilitating their implementation efforts. One participant described having time protected to conduct case reviews and said that their supervisors were, "very supportive of this as well. ...if I needed to go to them and say, 'hey, I need more time blocked to do these STORM reviews'...they would do that."

\section{Engaging}

The Engaging construct had a difference score of 1.8, with a CFIR mean rating from the bottom quartile of -0.5 and a CFIR rating from the 10 sites in the top quartile of 1.3. Although Engaging was rated 
moderately positively across all sites (overall mean rating $=.6$ ), the ability to form and work effectively with a team to perform case reviews clearly differed between sites with high vs. low mean CFIR ratings.

The Engaging construct was operationalized by asking about whether appropriate individuals were involved in the rollout and how available and engaged they were in establishing the process. Having a team in place was a strong facilitator for sites, where the addition of the STORM policy was often rolled into the existing process with minimal effort. For example, sites stated having a preexisting opioid prescribing safety program or team made it easy to implement the STORM policy. A staff member at one of the top quartile sites noted: "So...it had already been in the works... we had a lot of flow maps already written down. I think we had a charter in place. And, the STORM just became one more thing we were already...working on. It kind of took over our opioid safety reviews."

For these sites, the process was incremental and involved changes to existing processes rather than creation of new ones. One staff member said: "it was a little easier for us because we were already doing these things... so now we're in good shape and ...it was easy to just say...We have to change our note... this is what the note has to be called..." Although pre-existing teams had to adjust to a new method of review, they had the existing structure and skill to respond to the notice, making the demands on these sites quite different from sites without existing teams.

In sites without existing teams, a single individual or very small group might attempt to do the case reviews, often with mixed results, depending on the number of case reviews they were asked to complete. Consistent with the Engaging construct, the sheer effort of creating the team, including finding the right members, learning the needed skills, and developing a process to complete the reviews was an implementation barrier for some sites. One individual in a low quartile site noted: "we felt pressured and then we...also didn't know...how we were going to gather all that information and put it in the note, so that took a little bit of time. So, if we had all that worked out beforehand, it would have been a much easier, much simpler process. More effective."

A related challenge was team development. The policy notice recommended an interdisciplinary team, and over all interviews, 27 different roles were mentioned as participating in the case review process, with professional backgrounds varying from medical doctors and nurse practitioners to recreational therapists and podiatrists. The complexity of creating such an interdisciplinary team, in a large and sometimes siloed environment like the VA, was considerable. Participants described difficulty finding members to participate, challenges with identifying time to work on reviews together, and communication struggles between disciplines. Forming new teams often created an implementation barrier, as evidenced by the frustration from this interviewee: "....we've tried to partner with the suicide prevention people and they're overwhelmed too. They're like ... oh we can't take responsibility for that. Don't tag us onto the note. Don't tell us... Yeah, so it's like, well if not you, who?"

Another participant also noted a lack of role clarity around who should take responsibility for establishing and maintaining the team could sometimes be an issue, stating: "There is that push and pull, "This should 
be Mental Health's baby. No, this should be Primary Care's baby. No, this should be Pain Clinic's baby.' ...I think it should be an interdisciplinary team. "

Implementation climate

The Implementation climate construct had a difference score of 1.6, with a mean CFIR rating from the bottom quartile of-0.9, and a CFIR rating from the top quartile of 0.7 . This was the third highest difference score, tied with Networks and communications. However, the memos and research team discussion identified Implementation climate as an important indicator of the overall implementation at a site.

The construct was assessed by asking "How receptive are people in your organization to implementing the STORM Notice?" In low quartile sites, participants described organizational resistance: "The people in our organization unfortunately are not that interested to do new things. Unfortunately." Specific implementation climate barriers, as related by participants from two low quartile sites, included provider perception of burden and the difficulty of managing pain: "People are pretty resistant to implementing STORM, as well as most OSI initiatives, even those required by state law...because everybody feels like it is more work". Another stated: "look, a lot of people just hate dealing with pain. They don't know how. They don't like doing it. And, anything with opioids and Tramadol and now Lyrica makes it harder for them."

In a high quartile site, with a more receptive climate, one participant noted: "I think at first, just like with any new notice, (they) sort of, groaned a little bit and...dragged their feet but I think we're, we're good now. I think our facility is really receptive." Sites in the top quartile were more likely to have provided a positive context for implementing the STORM notice.

Available resources

The Available resources construct showed a difference of 1.3 in ratings between sites in the top (mean rating $=0.8$ ) vs. bottom (mean rating $=-0.5$ ) quartiles overall CFIR rating. Available resources is a composite of physical and time resources, which may have impacted the overall difference score. While most participants stated they had adequate space and equipment to carry out the reviews, many described a lack of staff and time for the work. Thus, while potentially not reflected in the overall difference ratings, insufficient time to carry out the reviews was the most pervasive barrier experienced across sites. This was reflected in direct questions about resources and indirectly mentioned frequently throughout the interviews. Staff with less flexibility in their schedule sometimes completed the reviews before their workday started or during their lunch break. Many simply stated that they "fit it in somehow" to their day. Although many described the importance of completing the work, in some cases this lack of time created resentment from staff.

Interviews in the low quartile sites demonstrated this concern, with one staff member stating: "...the problem is that we get zero dedicated time. And we've asked leadership several times, and it just falls on deaf ears so, no, we get zero dedicated time to do this." Many of those interviewed were adding this to an 
already full workload, as described by this individual: "I'm also a provider...I have a full patient load...And there's not really time to do all this." In a few cases, clinic time slots were blocked so providers could complete case reviews, but more typically, and still infrequently, they were only given time to attend the team meeting to discuss the reviews. When providers had flexibility in their schedule to complete the reviews, they described this as a facilitator.

Other CFIR constructs were barriers or facilitators in some cases, as described in Table 3, but were less pervasive or intense than those identified above. This is evident from the qualitative review as well as the percentage of positive ratings provided for each construct.

\section{Discussion}

In this evaluation of the randomized rollout of a policy requiring case reviews at VHA facilities, we identified key implementation barriers and facilitators, although there was no association between implementation barriers and facilitators and the arms of the study or facility characteristics. The predominant facilitators were strong and appropriate engagement, supportive leadership, and a positive implementation climate. Important barriers included lack of time to complete the case reviews and a perceived lack of evidence for the intervention. The mixed methods approach and use of joint displays is a novel approach that enabled the analysis of a large volume of qualitative data. The combination of ratings and coded data helped to develop a strong picture of the barriers and facilitators for this intervention.

The differing study arms appeared to have no effect on implementation barriers and facilitators. Having increased oversight and requiring sites that did not meet case review goals to complete action planning did not seem to impact how facilities implemented the notice. This finding replicates earlier work from the larger project indicating that oversight did not change the number or type of strategies used for the implementation [4]. The oversight could have been viewed by facilities as both positive and negative, since it included assistance from the national office, which perhaps confounded the effect of this contingency. The other randomization condition -altering the timing of requiring more case reviews also did not impact implementation barriers and facilitators. This is true even though some facilities experienced a large increase in the number of required case reviews prior to their interviews. Because facilities were not told in advance that an increase would occur, it is not surprising that this qualitative evaluation, which focused on how the process for completing the case reviews was developed, did not show differences between time frames. Because the interviewers were intentionally blinded to the condition of the interviewees, we did not ask specific questions about this increase, and it was seldom mentioned spontaneously.

We were surprised to find no association between implementation experience and the facility characteristics explored. It might be expected that facilities with higher levels of care (complexity) or more training (academic detailing) would develop different, perhaps better, approaches to this implementation. This difference has been demonstrated in other research, including work on following clinical practice 
guidelines at the VA, where less complex, Western, more rural sites, had less implementation of practice guidelines [12]. It seems possible that the high level of inter-disciplinary work required by this notice cut across those simple characteristics and favored a configuration of resources, leadership, climate and engaging to maximize positive implementation. This need for synergy in the implementation of complex innovations is also described by Rapp et al. [13].

We identified four CFIR constructs as facilitators: from the CFIR Process Domain, the Engaging construct, and from the Inner Setting Domain, (Readiness for Implementation sub-domain): Leadership engagement, Implementation climate, and Available resources. Two constructs were important barriers: also in the "Readiness for implementation" sub-domain, Access to knowledge and information and from the Process domain: Reflecting and evaluating. This constellation of barriers and facilitators suggests a lack of readiness for the implementation at many sites, which relates directly to Implementation climate. The concept of implementation climate was originated by Klein, Conn and Sorra, and is directly related to employee perceptions of how the innovation will be supported by leadership and supported with resources and training [14]. The complexity of the case review process further intensified the need for planning and preparation for this implementation. As Damschroder notes, the need for implementation planning that is clearly detailed and involves schedules and task assignments only increases with the complexity of the intervention [15].

Implementing the STORM case reviews required sites across the country with varying capacity to respond to a very specific and complex task: completing interdisciplinary case reviews and successfully logging completion of reviews using a note in the electronic medical record. The task required team creation and management, pain expertise, and technical skills in managing the case review note and understanding the STORM tool. Further, the task was charged to a group already stretched by clinical demands, and each site was asked to develop an individualized approach to completing the task, rather than being provided with specific tools and task assignments. While sites developed many different approaches to the task, some had greater resources and capacity to accomplish this, and some were simply exhausted and frustrated. Although recent work by Kim et al. points to the importance of heterogeneity in implementation efforts [16], the complexity of this innovation points to a need for greater task clarity and specificity. In addition, although the task was complex, the evaluation of the task was extremely simple, a correctly titled note in the medical record. Fidelity to this task was not evaluated, and some participants commented that there was likely to be great variation in the depth and quality of this note. Finally, as Greenhalgh et al. [17] stress, it is important to recognize the potential impact of sociopolitical context in which this implementation was taking place, as this time frame was a period of political stress and resource challenges for the VHA system, with calls for privatization and changes to allow outside providers to serve Veterans.

While this was a novel approach to evaluation with several notable findings, there were several limitations. First, although CFIR addresses a wide range of barriers and facilitators, only a limited number can be included in a given interview without becoming overwhelming, and it is possible that some important constructs were omitted. Second, 23 sites declined to participate in the qualitative interview.

Page $13 / 22$ 
While it is possible that participating facilities systematically differed from non-participants in unmeasured ways, leading to a selection bias, participating and non-participating facilities did not significantly differ on objective measures. Alternatively, a 45-minute interview may simply have been challenging for busy providers to accommodate. Third, the analytical strategy of comparing the sites with the top and bottom quartiles of overall CFIR scores may have masked important constructs that functioned in the moderately rated sites. However, we believe comparing the top and bottom quartile allows us to glean useful barriers and facilitators that clearly differentiated sites. Finally, an objective implementation measure was not used in any analyses. Although case review completion rate was the designated implementation measure for each site, mapping this measure on to the memo ratings proved unreliable due to the differing temporal relationship between the interviews and the completion rate, calculated quarterly. In addition, the measure did not quantify the actual number of case reviews completed but instead the proportion of reviews that were completed for a site over time. The outcomes of this policy initiative are being evaluated, and early evidence shows positive clinical outcomes for Veterans who are identified as at risk by the STORM dashboard. These Veterans are more likely to have a case review completed and to have risk mitigation strategies put in place [18].

In conclusion, this evaluation of a national randomized implementation identified key barriers and facilitators across multiple implementation sites. Although we found no difference in implementation barriers and facilitators across randomization arms, the evaluation demonstrated the value of strong, supportive leadership and climate, realistic expectations about time, and engaging the right people in creating a positive experience for implementation. Further, a perceived lack of training and accurate, well explained feedback were barriers to the process. In future large-scale, nationwide implementations it would be constructive to consider the overall readiness for implementation, including the development of strong implementation leadership [19]. In addition, more proactivity [20] and comprehensive training might help with improved adoption of an intervention of this complexity, as shown in much previous research $[5,17,21]$. Finally, the findings suggest the limits to using official policies promising low level consequences as a strategy to overcome implementation barriers.

\section{Conclusion}

In evaluating the randomized rollout of a policy requiring case reviews at VHA facilities, we did not find any association between study arms and implementation barriers and facilitators. Facilitators for the implementation of case reviews were strong engagement of appropriate individuals, engaged and supportive leadership, and a positive implementation climate. Lack of time to complete the case reviews and a perceived lack of evidence for the intervention were barriers. The evaluation used a mixed methods approach and joint displays, an innovative approach which enabled the analysis of a large volume of data.

\section{Abbreviations}

CFIR: Consolidated Framework for Implementation Research 
OTG: Opioid Therapy Guidelines

STORM: Stratification Tool for Opioid Risk Mitigation

VA: Veterans Affairs

VHA: Veterans Health Administration

\section{Declarations}

Ethics approval and consent to participate: The VA Pittsburgh Healthcare System approved this research study.

Consent for publication: Not applicable

Availability of data and materials: Please contact the corresponding author

Competing interests: The authors declare that they have no competing interests.

Funding: All the authors are funded by a grant from the Department of Veterans Affairs Health Services Research and Development Service: The STORM Implementation Program Evaluation (SDR 16-193).

Authors' contributions: SM, WG, GK, MS designed and conducted the interviews. SM, MC, MS, GK, WG, LH rated memos. HZ, MM, GK, SR and SM analyzed the data. SM drafted the manuscript. JH provided exceptional administrative support, and AG provided significant editing. All authors worked on study conception and design, interpretation of data, and critical review and approval of the manuscript.

Acknowledgements: We acknowledge that this work would not be possible without the cooperation and support of our partners in the Office of Mental Health and Suicide Prevention and in the HSR\&D-funded Partnered Evidence-Based Policy Resource Center. The contents of this paper are solely from the authors and do not represent the views of the Department of Veterans Affairs or the United States Government.

\section{References}

1. Oliva EM, Bowe T, Tavakoli S, et al. Development and applications of the Veterans Health Administration's Stratification Tool for Opioid Risk Mitigation (STORM) to improve opioid safety and prevent overdose and suicide. Psychol Serv. 2017;14(1):34-49.

2. Chinman M, Gellad WF, McCarthy S, et al. Protocol for evaluating the nationwide implementation of the VA Stratification Tool for Opioid Risk Management (STORM). Implement Sci. 2019;14(1):5.

3. Rogal SS, Chinman M, Gellad WF, et al. Tracking implementation strategies in the randomized rollout of a Veterans Affairs national opioid risk management initiative. Implement Sci. 2020;15(1):48. 
4. Minegishi T, Garrido MM, Pizer SD, et al. Effectiveness of policy and risk targeting for opioidrelated risk mitigation: a randomised programme evaluation with stepped-wedge design BMJ Open. 2018;8:e020097.

5. Damschroder LJ, Aron DC, Keith RE, Kirsh SR, Alexander JA, Lowery JC. Fostering implementation of health services research findings into practice: a consolidated framework for advancing implementation science. Implement Sci. 2009;4:50.

6. Damschroder LJ, Reardon CM, Sperber N, Robinson CH, Fickel JJ, Oddone EZ. Implementation evaluation of the Telephone Lifestyle Coaching (TLC) program: organizational factors associated with successful implementation. Transl Behav Med. 2017;7(2):233-241.

7. Damschroder LJ, Lowery JC. Evaluation of a large-scale weight management program using the consolidated framework for implementation research (CFIR). Implement Sci. 2013;8:51.

8. Gale RC, Wu J, Erhardt T, Bounthavong M, Reardon CM, Damschroder LJ et al. Comparison of rapid vs in-depth qualitative analytic methods from a process evaluation of academic detailing in the Veterans Health Administration. Implement Sci. 2019;14(1):11.

9. Forman J, Damschroder, L. Qualitative content analysis. In empirical methods for bioethics: A primer, 2007. Bingley, West Yorkshire, England:Emerald Group Publishing Limited;2007.

10. Campbell JL, Quincy C, Osserman J, Pedersen OK. Coding in-depth semistructured interviews: problems of unitization and intercoder reliability and agreement. SMR. 2013;42(3):294-320.

11. Guetterman TC, Fetters MD, Creswell JW. Integrating quantitative and qualitative results in health science mixed methods research through joint displays. Ann Fam Med. 2015;13(6):554-561.

12. Buscaglia AC, Paik MC, Lewis E, Trafton, JA. Baseline variation in use of VA/DOD clinical practice guideline recommended opioid prescribing practices across VA health care systems. Clinical J Pain. 2015;31(9):803-812.

13. Rapp CA, Etzel-Wise D, Marty D, Coffman M, Carlson, L, Asher D, Holter M et al. (2010). Barriers to evidence-based practice implementation: Results of a qualitative study. Community Mental Health J. 2010;46(2):112-118.

14. Klein KJ, Conn AB, Sorra JS. Implementing computerized technology: An organizational analysis. J Appl Psychol. 2001;86(5):811.

15. Damschroder LJ, Aron DC, Keith RE, Kirsh SR, Alexander JA, Lowery JC. Fostering implementation of health services research findings into practice: a consolidated framework for advancing implementation science. Implement Sci. 2009;4:50;extra file 4. 
16. Kim B, Sullivan JL, Ritchie MJ, Connolly SL, Drummond KL, Miller CJ et al. Comparing variations in implementation processes and influences across multiple sites: What works, for whom, and how? Psychiatry Res. 2020;283:112520.

17. Greenhalgh T, Glenn R, Fraser M, Bate P, and Kyriakidou O. Diffusion of innovations in service organizations: systematic review and recommendations. The Milbank Q. 2004;82(4):581-629.

18. Strombotne K, Legler A, Minegishi T, Trafton JA, Oliva EM, Lewis ET et al. Reducing adverse events from opioid prescriptions in the Veterans Health Administration: a stepped wedge cluster randomized controlled trial. Presented at: 2021 Annual Research Meeting of AcademyHealth;2021 Jun 14-17; virtual.

19. Bonham CA, Sommerfeld D, Willging C, Aarons GA. Organizational Factors Influencing Implementation of Evidence-Based Practices for Integrated Treatment in Behavioral Health Agencies. Psychiatry J. 2014;2014: 802983.

20. Birken SA, Lee SYD, Weiner BJ, Chin, MH, Schaefer, CT. Improving the effectiveness of health care innovation implementation: middle managers as change agents. Med Care Res Rev. 2013;70(1):2945 .

21. Phillips SD, Allred CA. Organizational management: What service providers are doing while researchers are disseminating interventions. JBHS\&R. 2006;33(2):156-175.

\section{Tables}

Table 1. Interview participant characteristics 


\begin{tabular}{|ll|}
\hline Participant characteristics & $\mathrm{N}=78$ \\
\hline Gender, $\mathrm{n} \%$ & \\
\hline Female & $45(58 \%)$ \\
\hline Male & $33(42 \%)$ \\
\hline & \\
\hline Training & \\
\hline Physician & $24(31 \%)$ \\
\hline Pharmacist & $28(36 \%)$ \\
\hline Psychologist or Social worker & $15(19 \%)$ \\
\hline NP or nurse & $11(14 \%)$ \\
\hline & \\
\hline Duration of VA Role & \\
\hline$<1$ year & $12(15 \%)$ \\
\hline $1-5$ years & $49(63 \%)$ \\
\hline $6-10$ years & $8(10 \%)$ \\
\hline$>10$ years & $12(15 \%)$ \\
\hline & $50(64 \%)$ \\
\hline Role in STORM & $24(31 \%)$ \\
\hline Lead & $4 \%)$ \\
\hline Team Member & \\
\hline No active role & \\
\hline
\end{tabular}

Table 2. CFIR ratings by randomization arm for 39 facilities 
Average Percentage of Positive Ratings

\begin{tabular}{|c|c|c|c|c|}
\hline \multirow{2}{*}{$\begin{array}{l}\text { All } \\
\text { Arms }\end{array}$} & Arm 1 & Arm 2 & Arm 3 & Arm 4 \\
\hline & $\begin{array}{l}\text { No } \\
\text { oversight } \\
\text { and } \\
\text { early } \\
\text { increase, } \\
\text { Mean } \\
\text { (SD) }\end{array}$ & $\begin{array}{l}\text { No } \\
\text { oversight } \\
\text { and late } \\
\text { increase, } \\
\text { Mean } \\
\text { (SD) }\end{array}$ & $\begin{array}{l}\text { Oversight } \\
\text { and } \\
\text { early } \\
\text { increase, } \\
\text { Mean } \\
\text { (SD) }\end{array}$ & $\begin{array}{l}\text { Oversight } \\
\text { and late } \\
\text { increase, } \\
\text { Mean (SD) }\end{array}$ \\
\hline$N=39$ & $\mathrm{n}=10$ & $\mathrm{n}=10$ & $n=10$ & $n=9$ \\
\hline
\end{tabular}

Engaging

0.6
$(1.0)$

$0.6(1.0)$

$0.9(0.9)$

$0.5(1.1)$

$0.4(1.0)$

0.71

Planning

0.5

(1.1)

$0.4(0.8)$

$0.9(1.0)$

$0.5(1.2)$

$0.3(1.3)$

0.66

(1.1)

Reflecting Evaluating

$-0.4$

$-0.8(1.1)$

$-0.5(0.7)$

$-0.2(1.0)$

$0.1(0.9)$

0.17

(1.0)

Tension for Change

0.7

$0.7(0.7)$

$0.8(0.4)$

$0.7(0.5)$

$0.7(0.5)$

0.91

(0.5)

Implementation

Climate

Compatibility
-0.1
$(1.1)$

$-0.1(0.7)$

$-0.2(1.2)$

0.71
$-0.2(1.2) \quad 0.3(1.1)$

$0.2(1.2) \quad 0.3(1.1)$

$-0.2(1.2)$

$0.1(1.0)$

$0.2(1.0)$

$-0.1(1.1)$

0.84
Structural

Characteristics

Networks

Communications
0.5
$(0.9)$
-0.2
$(0.9)$

$-0.3(0.9)$

$-0.1(0.9)$

$0.2(0.9)$

$-0.6(0.7)$

0.25 
Leadership Engagement
$0.3 \quad 0.0(1.4) \quad 0.3(0.8)$

(1.1)
$0.1(1.1)$

$0.7(1.0)$

0.64
Relative Priority

0.7

(0.9)

$0.2(1.2) \quad 0.8(0.4)$

$0.9(0.6)$

$1.0(0.9)$

0.27

Available Resources

0.1

$0.0(1.1)$

$-0.3(0.8)$

$0.3(1.0)$

$0.4(1.1)$

0.56

(1.0)

Access to Knowledge Information

0.1
$(0.9)$

$-0.2(0.8) \quad 0.0(1.1)$

$0.2(1.0)$

$0.4(0.7)$

0.42

(0.9)

Evidence

-0.4
$(0.8)$

$-0.2(0.9) \quad-0.4(0.8)$

$-0.6(0.8)$

$-0.5(0.8)$

0.78

(0.8)

Relative Advantage

0.2
$(0.7)$

$0.0(0.7) \quad 0.2(0.7)$

$0.1(0.7)$

$0.4(0.9)$

0.66

Patient Needs

Resources

Peer Pressure
0.3

(0.7)
$0.5(0.5)$

$-0.2(0.8)$

$0.5(0.5)$

$0.6(0.7)$

0.09
Total CFIR Score (mean of 16 construct ratings)
$0.1 \quad 0.0(0.8)$

(0.7)
0.2

(0.5)

$0.1(0.6)$

$0.2(0.4)$

$0.2(0.4)$

$0.3(0.5)$

0.77

0.79

$0.1(0.9) \quad 0.3(0.7)$ 
Table 3 to be inserted here (Table 3. Joint display of quantitative and qualitative data about STORM barriers and facilitators)

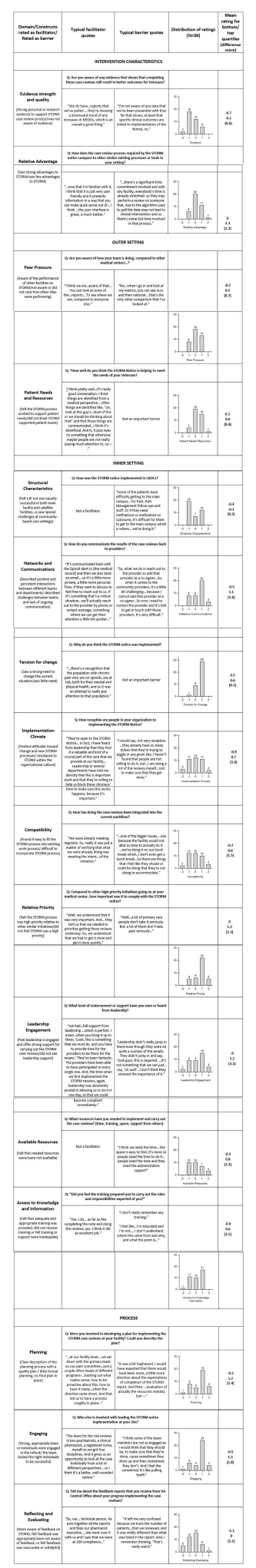

\section{Supplementary Files}


This is a list of supplementary files associated with this preprint. Click to download.

- Additionalfile2STORM.docx

- StaRIchecklistSTORM.docx

- additionalfilenumberone.docx 